\title{
Adaptive yielding scheme for link scheduling in OFDM-based synchronous device-to-device (D2D) communication system
}

\author{
Chung G Kang ${ }^{3 *}$, Jin W Kim ${ }^{1}$, Hye J Kang ${ }^{3}$ and Minjoong Rim²
}

\begin{abstract}
Compared to asynchronous contention-based random access, e.g., carrier sensing multiple access, synchronous and distributed link scheduling for orthogonal frequency-division multiplexing (OFDM) systems is a viable solution to improve system throughput for device-to-device (D2D) ad hoc network. In particular, spatial spectral efficiency can be improved by scheduling as many concurrent D2D links necessary to satisfy individual signal-to-interference ratio (SIR) requirements. In this paper, we propose an adaptive yielding mechanism that can further improve the spatial spectral efficiency by allowing for more concurrent D2D links whenever more interference can be accepted, e.g., when the instantaneous bandwidth efficiency requirement is less than the current link capacity. Even if the system throughput varies with the link density, it is shown that the average system throughput can be significantly improved by the proposed yielding mechanism.
\end{abstract}

\section{Introduction}

As opposed to the cellular systems which have been designed to support the mobile station throughout of the rather wide-area coverage, there have been various types of schemes or systems, including Wi-Fi Direct or Bluetooth, which establish links between nearby devices without resorting to an associated access point [1-3]. In the recent advance in cellular systems, such a device-to-device (D2D) communication scheme has been considered as a means of device-centric mobile social network service (SNS), which discovers the proximity devices and then connect them over the direct link [4]. In general, it can be implemented either as inband or outband D2D [5]. In the inband D2D type, the cellular spectrum is used for both cellular and D2D links. As the licensed spectrum can be fully controllable, quality of service (QoS) provision is ensured in the inband D2D type. Inband D2D communication can be further divided into underlay and overlay scenarios [5]. In this paper, we consider the overlay scenario, in which the D2D links are allocated to the dedicated cellular resources, thus incurring no co-channel

*Correspondence: ccgkang@korea.ac.k

${ }^{3}$ School of Electrical Engineering, Korea University, Seoul 136-701, South Korea Full list of author information is available at the end of the article interference between the cellular and D2D links. The obvious advantage of D2D communication in this scenario is that radio resources can be reused by supporting multiple D2D communication pairs at the same time if any, without resorting to macro-cell links [6-12]. In order to improve the spectral efficiency in the overlay D2D scenario, the direct communication can be either assisted by the base station (BS), which involves scheduling the resource for D2D link. However, D2D communication can be autonomous for the outband overlay scenario, in which D2D links can be scheduled without any centralized assistance by the base station. Once a dedicated spectrum is set aside for D2D communication in the overlay scenario, the idea is that D2D communication in the cellular system would turn it into a peer-to-peer ad hoc network. Then, we need to develop a distributed link scheduling protocol that can improve the spectral efficiency while spatially reusing the radio resource among the concurrent D2D links. One particular example in this scenario is the Qualcomm FlashLinQ system [13], which will be focused in this paper. A distributed type of D2D communication will be useful by connecting the handsets with each other in the cellular system when a base station is lost or the handsets are out of coverage, e.g., during an emergency situation. For example, the IEEE 802.16n and IEEE

\section{Springer}

(C) 2014 Kang et al.; licensee Springer. This is an Open Access article distributed under the terms of the Creative Commons Attribution License (http://creativecommons.org/licenses/by/4.0), which permits unrestricted use, distribution, and reproduction in any medium, provided the original work is properly credited. 
802.16.1 standards have been specified for public safety or Public Protection and Disaster Relief (PPDR) applications $[14,15]$. Recently, the same design objective has been considered by employing a new concept of informationtheoretic independent sets (ITIS), which may achieve a theoretical upper bound on the D2D capacity [16]. However, the underlying assumption behind the performance in [16] is that every transmitter knows the link scheduling status of all other links a priori, which cannot be implemented in practice.

On the other hand, new application services, including mobile advertisement, mobile social network service (SNS), mobile content sharing, and group data communication, can be developed if one mobile device discovers other nearby devices and D2D links are established. In particular, an obvious advantage of D2D communication in the orthogonal frequency-division multiplexing (OFDM)-based cellular system is extending coverage, such as that in D2D communication between handsets in the different cells, by concentrating the carrier power into a subset of the subcarrier, which facilitates different application services. This might not be possible in the existing local area networks (LANs) or personal area networks (PANs), simply due to the limited coverage. However, a distributed nature in their collision-avoidance type of access control mechanism is still similar to that in the peer-to-peer ad hoc networks.

A D2D communication system must deal with two different functionalities: discovery and communication. Consider multiple D2D mobile devices randomly distributed in a system. From a communication perspective in this system, the overall system throughput is one of the most important performance factors, as in all other networks. Link scheduling is required to establish the links for the multiple D2D pairs at the same time under QoS constraints while maximizing the overall system throughput. In particular, an OFDM-based D2D ad hoc network employs a synchronous and distributed medium access control protocol which controls the admissible interference with respect to signal-to-interference ratio (SIR) and outperforms the conventional carrier sensing multiple access scheme [13]. In order to guarantee the received SIR in all links, it executes the yielding procedures with the fixed SIR thresholds in both the transmitter and receiver. In this paper, we propose a new type of link scheduling scheme that can eliminate the inefficiency associated with distributed scheduling in a synchronous D2D ad hoc network and demonstrate its performance gain over the existing scheme. It allows for reusing other links spatially within the permissible range while consuming the link capacity only as much as enough to satisfy the required bandwidth efficiency for each link. Furthermore, it allows for a data rate fall-back when the traffic load drops, e.g., a transmission buffer becomes less congestive, which can maximize the spatial reuse efficiency adaptively with the traffic load and link distribution. In addition to improvement in the system throughput, these features enhance the fairness among the links, attributing to improving the average throughput of the low-priority access users, as opposed to the FlashLinQ system in which some users may always suffer from the lower access priority in each frame.

In Section 2, we first describe a basic model and the existing yielding scheme for link scheduling in the OFDMbased D2D communication system. A concept of the proposed adaptive yielding scheme is presented in Section 3. The specific procedure to implement the proposed concept is given in Section 4. Its performance is evaluated by system-level simulation in Section 5 . Finally, Section 6 presents the concluding remarks.

\section{Yielding mechanism for OFDM-based synchronous D2D communication}

As our proposed scheme is strictly based on the existing system, e.g., FlashLinQ [13], we describe a common system model for link scheduling in the OFDM-based D2D communication system. In fact, the frame and signaling structures in the specification of FlashLinQ are provided to serve as a common system model for our discussion. Furthermore, since our scheme is an improved version of the link-scheduling mechanism in FlashLinQ, the procedure of the existing one is detailed in this section.

\subsection{System model}

A unique connection identifier (CID) is assigned to an individual D2D link. For example, 112 unique numbers (1 through 112) are assigned as CIDs in FlashLinQ [13], where two single tones, one for the transmitter and the other for the receiver, are uniquely mapped to each of the CIDs. Twenty-eight OFDM tones are used for signaling over four OFDM symbols, corresponding to a total of 112 tones. Each of these orthogonal tones will be used for a corresponding D2D link without any interference from any other tones. The sets of tones to be used by the transmitters and receivers are defined as the Tx block and Rx block, respectively (see Figure 1). The tones in the Tx block are used for indicating a request to schedule by transmitting at a tone that is uniquely mapped to one's own CID. The indication signal over the Tx block is referred to as an RTS (Request to Send). This particular signal is similar to RTS transmission in the IEEE 802.11 specification, and it will be used to ensure that the receiver is going to accept its transmission. If it decides not to yield the current connection, a designated tone in the Rx block is employed as a CTS (Clear to Send) signal, which allows for estimating the SIR in the transmitter. Only when both transmitter and receiver decide not to yield their connection, then their D2D communication 


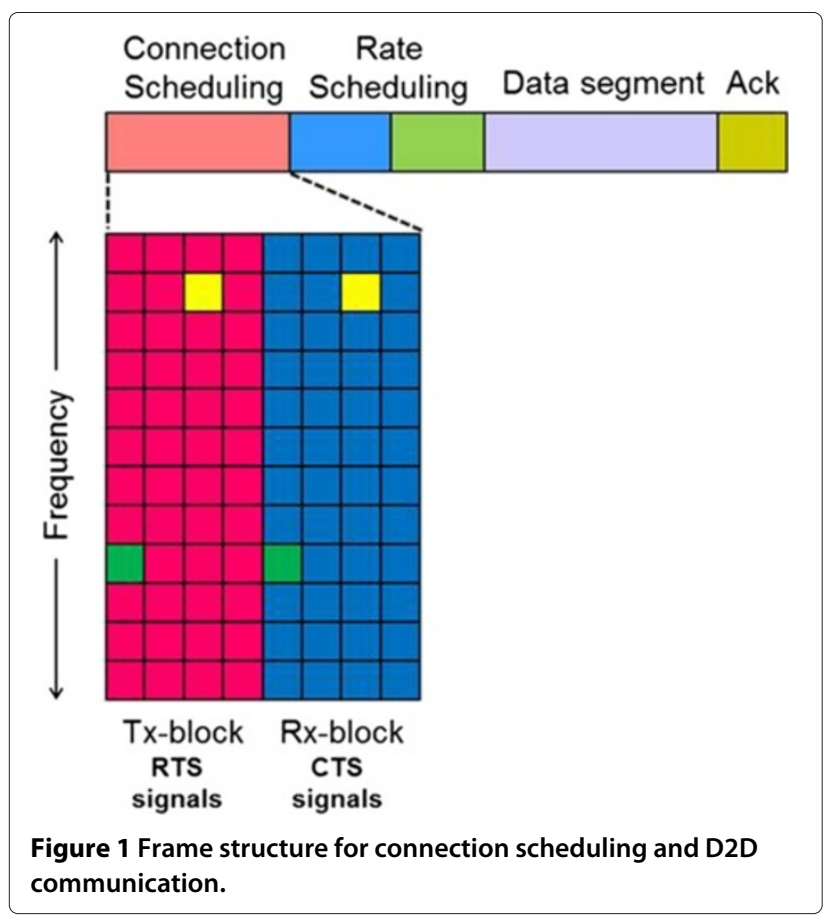

can be initiated. Figure 1 shows the frame structure, illustrating the Tx block and Rx block for connection scheduling.

\subsection{Yielding procedure for connection scheduling}

D2D communication requires a distributed link scheduling procedure, which establishes a D2D link without resorting to any centralized control. One particular example is a handshaking mechanism between the Tx and Rx nodes over the D2D link for CSMA-CA protocol, which employs RTS and CTS control frames in the IEEE 802.11 Wireless LAN specification [17]. More specifically, a Tx node of the D2D link transmits an RTS frame and a corresponding $\mathrm{Rx}$ node responds with a CTS frame, clearing up its neighbor nodes that would be interfering with the established D2D link. The RTS-CTS handshaking procedure eliminates the hidden interfering nodes, which cannot be directly identified by the Tx node of the D2D link. Figure 2a illustrates the RTS-CTS handshaking procedure in the IEEE 802.11 Wireless LAN specification, in which any node within the range (CTS circle for link A$B)$ that can receive the CTS frame transmitted by node A over link A-B refrains from transmitting. The number of D2D links that can be reused is determined by the radius of the CTS circle. As RTS and CTS control frames can be transmitted at any time, without any synchronization mechanism for RTS-CTS handshaking, medium access control (MAC) in the IEEE 802.11 Wireless LAN specification corresponds to the asynchronous protocol. As the Tx-Rx distance is not known in general, RTS and CTS circles must be large enough in this particular handshaking procedure, which may incur inefficiency of the spatial reuse. Even though there have been various attempts to handle such shortcomings [18-23], they are not very successful in taking co-channel interference into account for connection scheduling.

In an OFDM-based synchronous D2D communication system, such as Qualcomm FlashLinQ, node-specific OFDM tones can be used to measure co-channel interference either directly or indirectly among the D2D nodes. In that case, radii of RTS and CTS circles can be determined so as to maximize the spatial reuse efficiency. Figure $2 b$ shows the RTS and CTS circles established for FlashLinQ, illustrating a situation of spatially denser connection scheduling. In the sequel, we will detail the connection scheduling procedure for FlashLinQ.

Figure 3 presents a simple D2D link model in which a D2D link $C-D$ is to be scheduled while a high-priority link already has been established between nodes $A$ and $B$. Note that $\left\{A^{\prime}{ }_{i}\right\}_{i=1}^{N}$ in Figure 3 represents the transmitters for high-priority links, which already have been scheduled, i.e., ones that are simultaneously transmitting with link $A-B$. This particular illustrative model will be
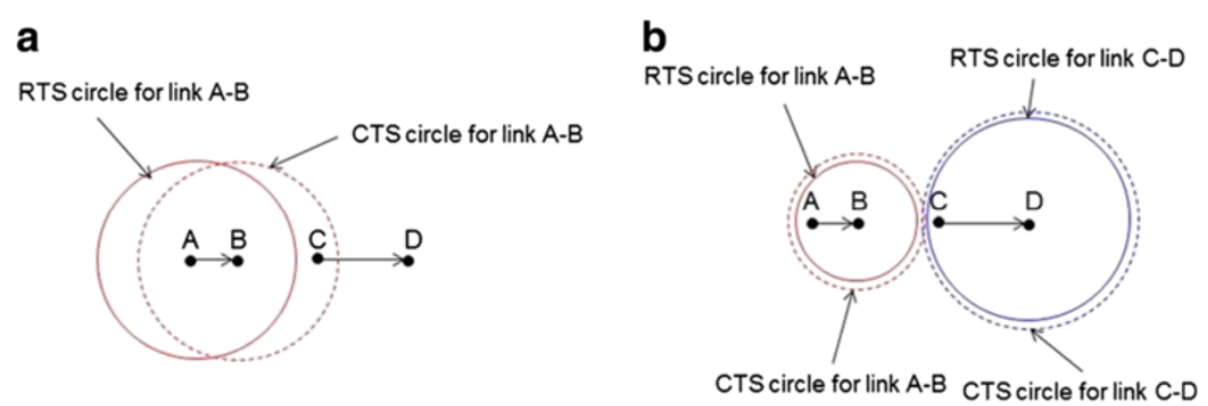

Figure 2 RTS-CTS handshaking: IEEE $\mathbf{8 0 2 . 1 1}$ specification vs. FlashLinQ. (a) RTS-CTS handshaking in IEEE 802.11 specification. (b) RTS-CTS handshaking in FlashLinQ. 


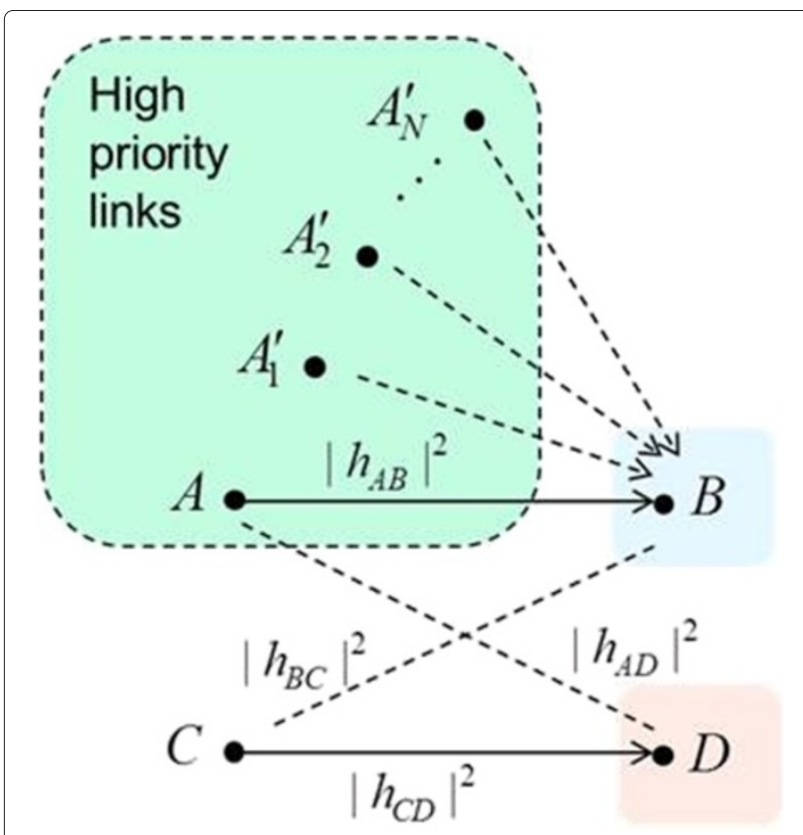

Figure 3 D2D Link model for connection scheduling.

referenced to explain a basic operational procedure for connection scheduling under consideration. In Figure 3, $h_{X Y}$ represents a channel gain between nodes $X$ and $Y$. The receiver is to give up D2D communication when its own received signal is not large enough compared to the received signal from the higher-priority links. In other words, the D2D receiver gives up the corresponding link connection when the received SIR is below the given threshold $\eta_{R X}$, which is referred to as Rx yielding. All transmitters are to send RTSs by using the designated tones with the fixed level of power in the Tx block, which allows for a receiver to measure the corresponding SIR. If the measured SIR exceeds the given threshold $\eta_{R X}$, the receiver responds with CTS using the Rx block. Otherwise, it does not respond, which implicitly indicates $\mathrm{Rx}$ yielding.

Referring to the D2D link $C-D$, which is to be scheduled when the high-priority link $A-B$ already has been established in Figure 3, Rx node $D$ receives power of $P_{A}\left|h_{A D}\right|^{2}$ as interference when Tx node $A$ transmits at power of $P_{A}$. In case that SIR measured at Rx node $D$ is below the $\mathrm{Rx}$ yielding threshold, link $C-D$ must give up the connection so as to avoid unnecessary transmission subject to an outage over the link $C$ - D. More specifically, the $\mathrm{Rx}$ node $D$ must yield $\mathrm{D} 2 \mathrm{D}$ communication if the following condition is met ( $\mathrm{Rx}$ yielding condition):

$$
\frac{P_{C}\left|h_{C D}\right|^{2}}{P_{A}\left|h_{A D}\right|^{2}}<\eta_{R X}
$$

where $\eta_{R X}$ is referred to as an Rx yielding threshold. If (1) is satisfied, then Rx node $D$ does not transmit a CTS in response to an RTS, which indicates Rx yielding.

Meanwhile, the low-priority link can be established as long as its interference toward the high-priority link is not too large. In other words, the Tx node of the lowpriority link must yield its D2D connection to protect the high-priority link if the potential link would incur interference toward the high-priority link. When Tx node $A$ of the high-priority link transmits RTS with power of $P_{A}$ in Figure $3, \operatorname{Rx}$ node $B$ receives it with power of $P_{A}\left|h_{A B}\right|^{2}$. Meanwhile, if Tx node $C$ transmits with power of $P_{C}$, it would incur interference to Rx node $B$ of the high-priority link. In case that SIR measured at Rx node $B$ is below the given threshold $\eta_{T X}$, Tx node $C$ of the low-priority link must yield to protect the highpriority link, which is referred to as Tx yielding. More specifically, the Tx node $C$ must yield D2D communication if the following condition is met (Tx yielding condition):

$$
\frac{P_{A}\left|h_{A B}\right|^{2}}{P_{C}\left|h_{B C}\right|^{2}}<\eta_{T X}
$$

where $\eta_{T X}$ is referred to as a Tx yielding threshold. In order to check the Tx yielding condition in (2), Tx node $C$ must be able to estimate the value of $P_{A}\left|h_{A B}\right|^{2} /\left|h_{B C}\right|^{2}$. Toward this end, Rx node $B$ of the high-priority link transmits an inverse power echo, which corresponds to the inverse of the received power from Tx node $A$. Then, Tx node $C$ receives a power of $r_{p}=\left|h_{B C}\right|^{2} /\left(P_{A}\left|h_{A B}\right|^{2}\right)$, which includes the channel gain $h_{B C}$ between Tx node $C$ and $\mathrm{Rx}$ node $B$. After Rx node $C$ receives inverse power echo, the left-hand side of (2) can be estimated by $1 /\left(r_{p} \cdot P_{C}\right)$, which allows for the determination of whether Tx node $C$ must yield or not.

\section{Adaptive yielding mechanism: overview}

\subsection{Motivation}

The key idea of improving the throughput over the existing link scheduling scheme in this paper is to allow the low-priority link to be scheduled within a range of the allowable interference by minimizing the unnecessary transmission opportunities of the high-priority links. A notion of unnecessary transmission is viewed from the two different points. One point is that a link can reduce its own transmission rate by accepting additional interference when a packet waiting in the transmit buffer is too short to fill up the current slot in the course of its link scheduling. In other words, a link capacity is set to the minimum data rate that is required to meet its own demand in the current slot while accepting more interference so as to allow the low-priority links to be scheduled simultaneously. As additional interference is allowed only as long as the minimum capacity required for the high- 


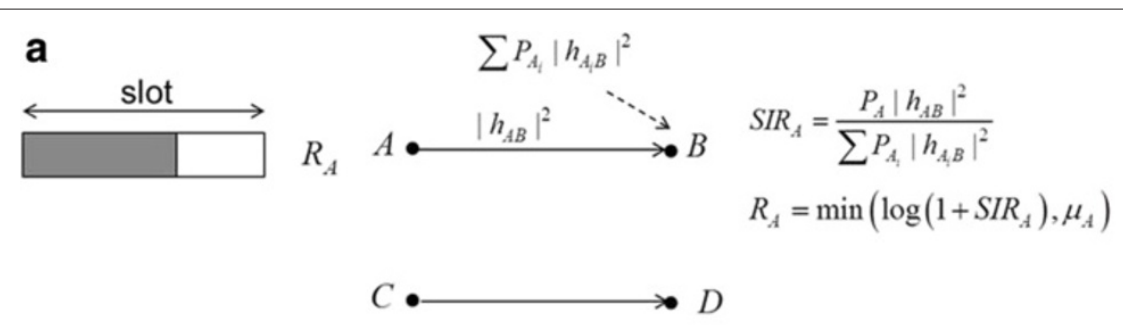

b

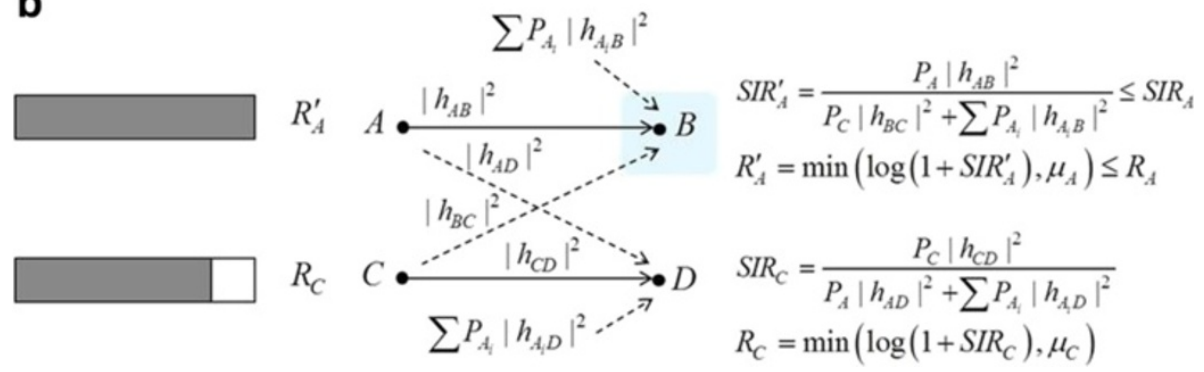

Figure 4 Throughput enhancement by effect of Tx fallback: illustration. (a) No Tx fallback. (b) Tx fallback.

priority link is guaranteed, the current sharing process is referred to as conservative yielding. That is, a conservative yielding mechanism of the high-priority link allows for the low-priority link to be scheduled opportunistically, only whenever the high-priority link can reduce its own data rate for the packet to transmit in the current slot. In spite of conservative yielding, however, a receiver of the low-priority links may still have to yield its transmission (i.e., Rx yielding) due to interference caused by the high-priority links. For the conservative yielding mechanism to be effective from the first viewpoint, therefore, a transmitter of the high-priority link must give up its transmission or reduce its transmit power for the lowpriority link not to perform $\mathrm{Rx}$ yielding. Toward this end, the second viewpoint of minimizing the unnecessary transmission of the high-priority link is to reduce its transmission power as long as the total size of the packets waiting in the buffer of the high-priority link is below the given threshold. As the high-priority link reduces its own transmit power, it may be faced with $\mathrm{Rx}$ yielding. Since this particular yielding process involves with sacrificing the high-priority link when its capacity is not immediately required, e.g., in terms of its buffer status, it is referred to as generous yielding. Note that the aforementioned conservative yielding becomes effective only when it is combined with generous yielding. It is due to the fact that the low-priority link can be scheduled only when it is not subject to both Rx yielding and Tx yielding at the same time. In other words, even if the high-priority link is not subject to Tx yielding by conservative yielding of the highpriority link, its receiver still may have to yield due to interference from the links of the higher priority, making the conservative yielding mechanism useless. Therefore, our proposed design aims at combining both conservative and generous yielding mechanisms so tightly that the system throughput performance can be enhanced by minimizing unnecessary transmission of the high-priority links.

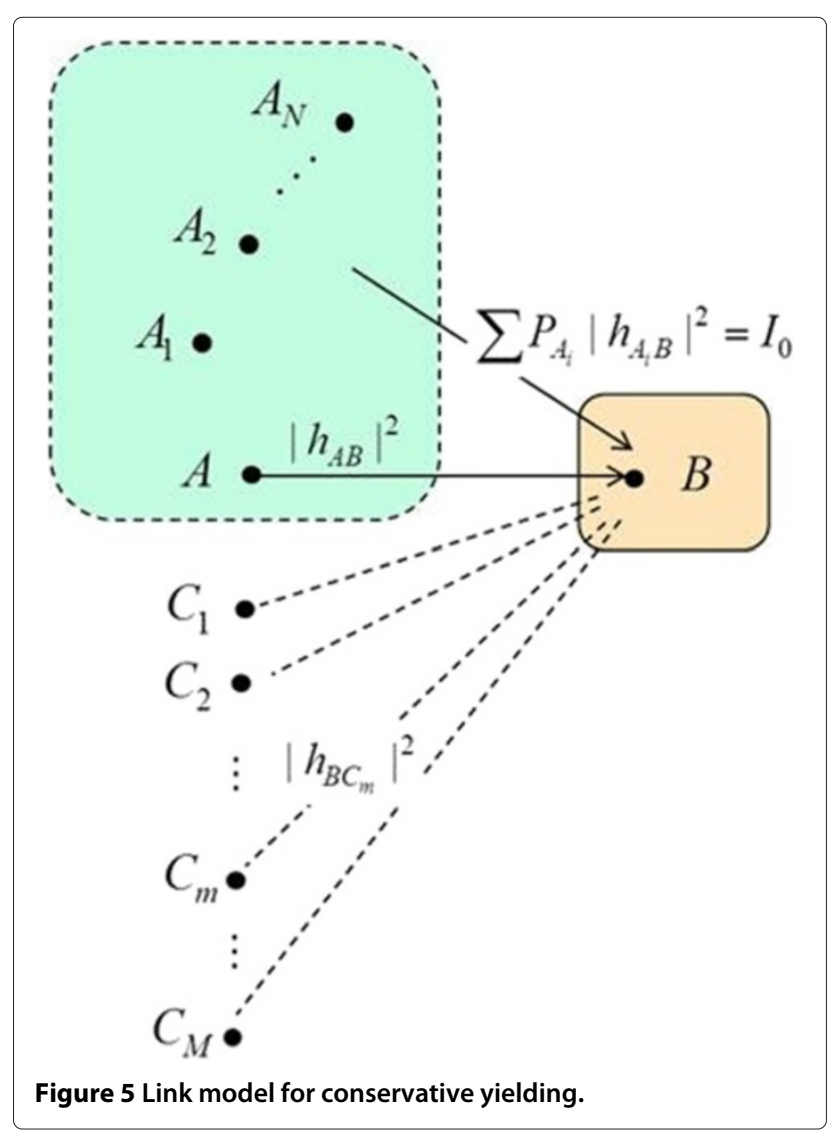




\subsection{Throughput enhancement with Tx fallback-based adaptive yielding mechanism}

The D2D link in Figure 3 can still be referred to in order to investigate the throughput enhancement effect of the proposed adaptive yielding mechanism. Let the slot length and packet length be $T$ (seconds) and $L$ (bytes), respectively. Furthermore, let $Q$ denote the number of bits for the awaiting packets in the buffer. Without loss of generality, channel bandwidth is normalized as $W=1 \mathrm{~Hz}$ for simplicity of presentation. Let $\mu_{A}$ be the capacity required to handle the packet waiting in the buffer at Tx node $A$, which is given as $\mu_{A}=Q / T$ (bits/s). Meanwhile, assume that the low-priority link $C$ - $D$ yields to the high-priority links by taking their interference into account, as illustrated in Figure 3. Then, the received SIR over the link $A-B$ is given by $S I R_{A}=\frac{P_{A}\left|h_{A B}\right|^{2}}{\sum P_{A_{i}}\left|h_{A_{i} B}\right|^{2}}$, which corresponds to the channel capacity of $\log \left(1+S I R_{A}\right)$ (bits/s).

Figure $4 \mathrm{a}$ illustrates a situation where the required capacity $\mu_{A}$ is smaller than the channel capacity $\log \left(1+S I R_{A}\right)$. In this case, an effective channel capacity is governed by $R_{A}=\min \left\{\log \left(1+S I R_{A}\right), \mu_{A}\right\}$. It implies that $S I R_{A}$ can be reduced just enough to support $\mu_{A}$ while allowing for additional interference by accepting

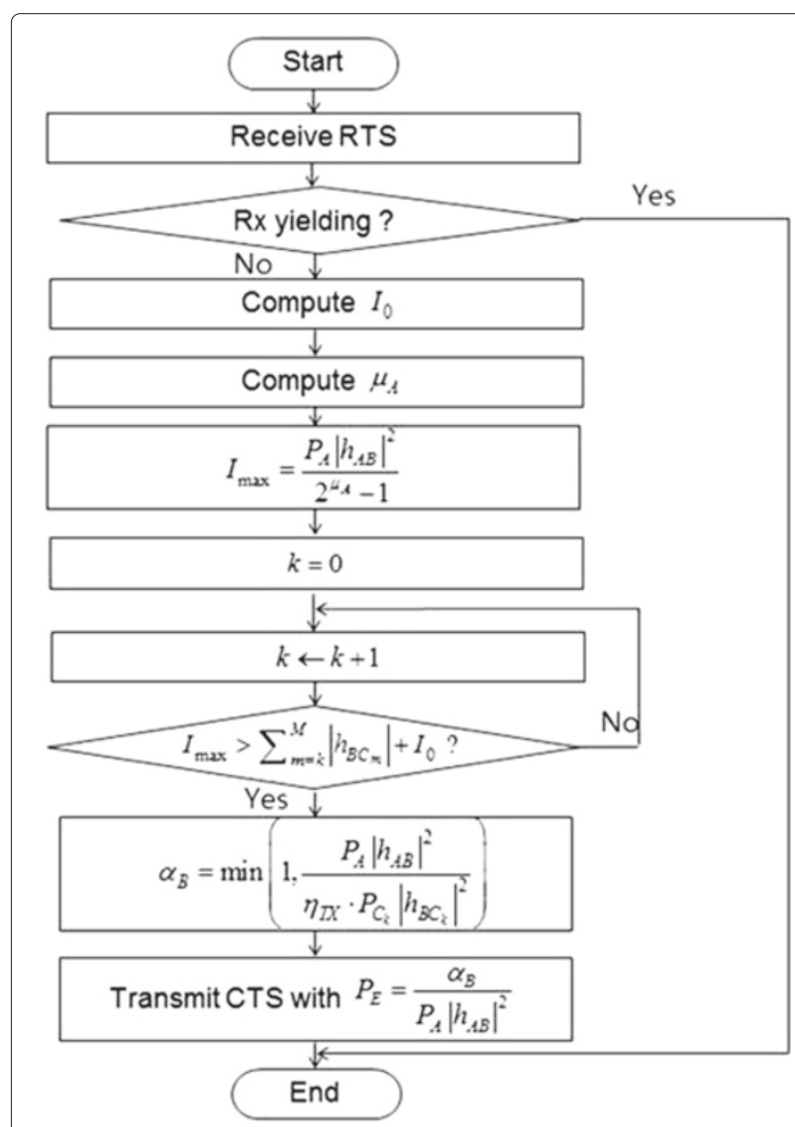

Figure 6 CTS power control for conservative yielding: flowchart. the low-priority link. In other words, Tx node $A$ can fall back into the lower data rate, as illustrated in Figure 4b. Then, additional interference would change the received SIR over the link $A-B$ to $\operatorname{SIR}_{A}^{\prime}=\frac{P_{A}\left|h_{A B}\right|^{2}}{P_{C}\left|h_{B C}\right|^{2}+\sum P_{A_{i}}\left|h_{A_{i} B}\right|^{2}}$, and thus, the effective channel capacity is given as $R_{A}^{\prime}=$ $\min \left(\log \left(1+S I R_{A}^{\prime}\right), \mu_{A}\right)$. Note that $\operatorname{SIR}_{A}^{\prime} \leq S I R_{A}$ and $R_{A}^{\prime} \leq R_{A}$. In the case that the low-priority link $C$ $D$ requires a capacity of $\mu_{C}$, its effective capacity is given as $R_{C}=\min \left(\log \left(1+S I R_{C}\right), \mu_{C}\right)$ where $S I R_{C}=$ $\frac{P_{C}\left|h_{C D}\right|^{2}}{P_{A}\left|h_{A D}\right|^{2}+\sum P_{A_{i}}\left|h_{A_{i} D}\right|^{2}}$. Therefore, if $R_{A}^{\prime}+R_{C}>R_{A}$, the overall system throughput is enhanced by falling back the data rate of the high-priority link from $R_{A}$ to $R_{A}^{\prime}\left(R_{A}>R_{A}^{\prime}\right)$. In other words, a Tx fallback mode becomes useful as long as the additional throughput of the low-priority links is larger than the high-priority throughput reduced by accepting the low-priority links. In the following section, we present how an adaptive yielding mechanism can be implemented along with the Tx fallback mechanism.

\section{Implementation of adaptive yielding}

\subsection{CTS power control for conservative yielding}

As analyzed in Section 3, the low-priority links can be accepted within an admissible range of additional

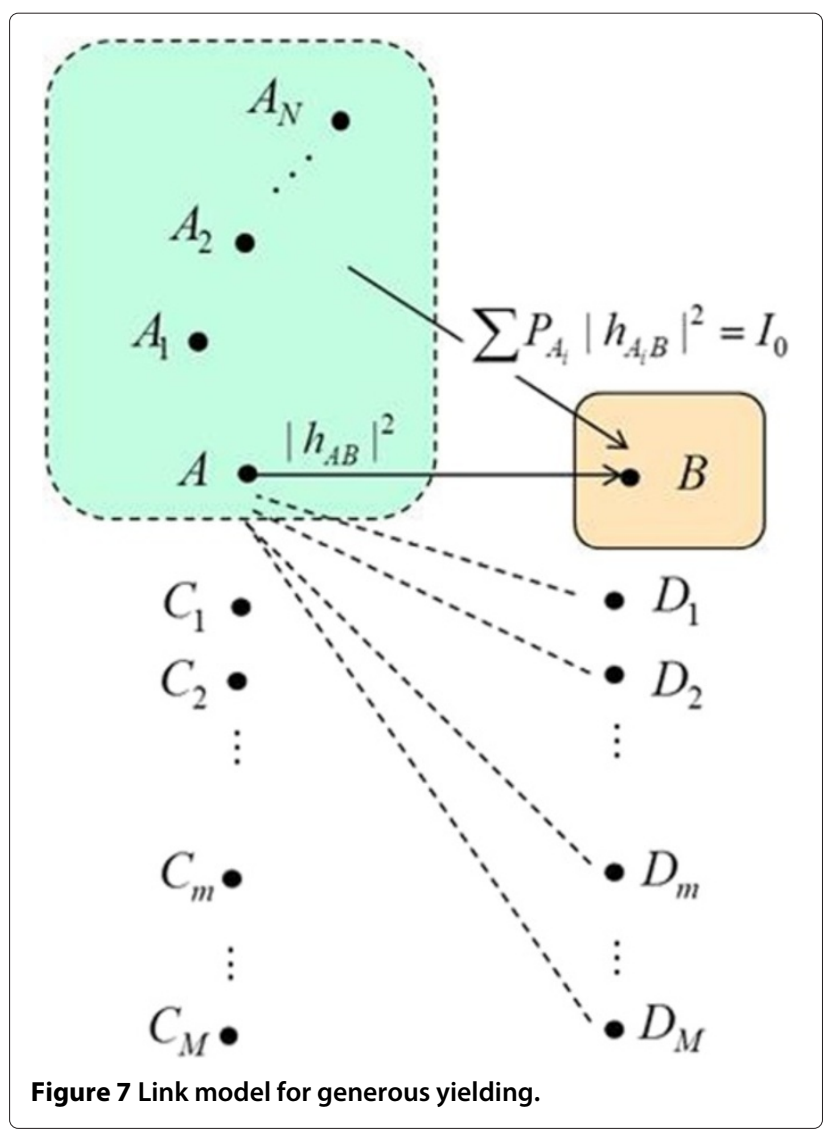




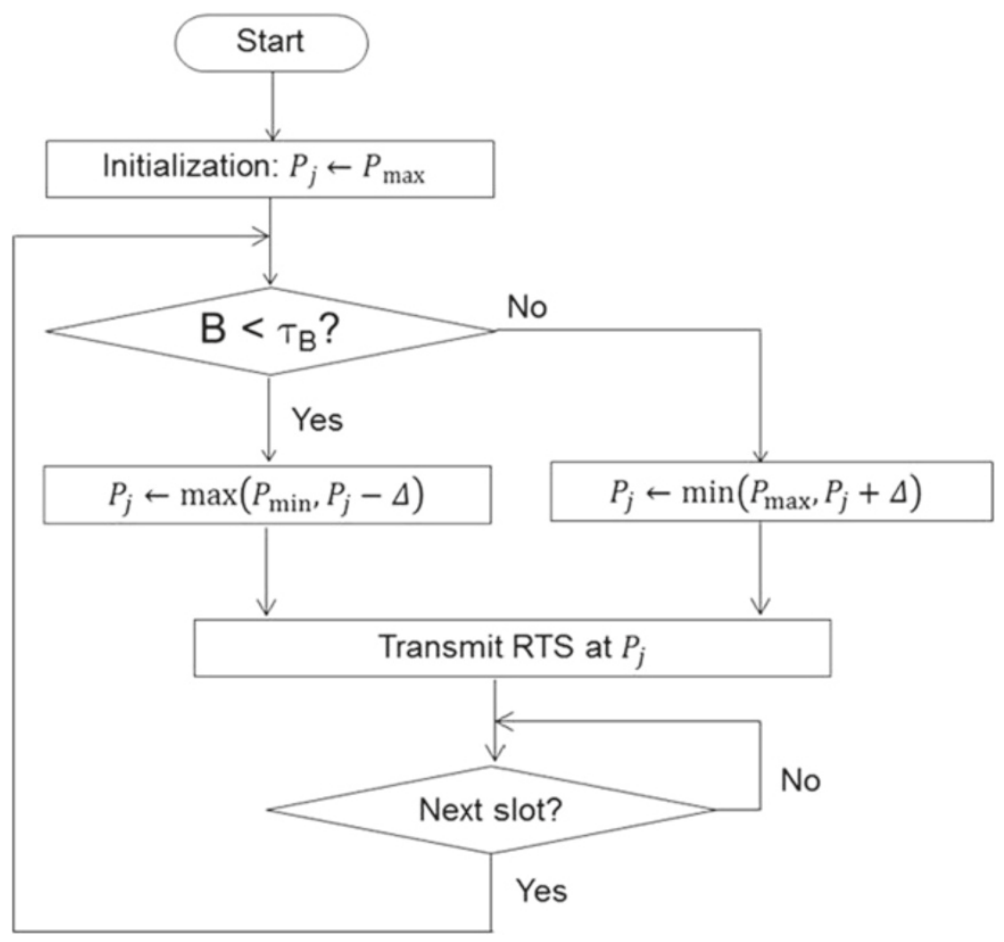

Figure 8 RTS power control for generous yielding.

interference. Toward this end, the additional allowable interference in the Rx node of the low-priority link must be determined (referring to $\mathrm{Rx}$ node $B$ in Figure 5) to derive a power level of CTS that allows its Tx node within the tolerable range of interference not to yield to the highpriority link. In other words, CTS power must be derived in the Rx node of the high-priority link for its conservative yielding. We will detail the CTS power control procedure associated with conservative yielding.

As illustrated in Figure 5, assume that there exists $M$ low-priority links with respect to a high-priority link $A$ $B$, which requires a bandwidth efficiency of $\mu_{A}$ (bps). As discussed in the previous section, $\mu_{A}$ is determined by the packet length or the number of transmit bits in the current slot. From the viewpoint of $\operatorname{Rx}$ node $B$, the low-priority links incur different levels of interference depending on their positions, i.e. with the different channel gains $\left\{h_{B C_{1}}, h_{B C_{2}}, \cdots, h_{B C_{M}}\right\}$ as shown in Figure 5 $\left(\left|h_{B C_{1}}\right|^{2} \geq\left|h_{B C_{2}}\right|^{2} \geq \cdots \geq\left|h_{B C_{M}}\right|^{2}\right)$. In order to determine the admissible links for Rx node $B$, ones with lower interference must be taken into consideration. Toward this end, assume that $(M-m+1)$ low-priority links are accepted for link scheduling, starting from the $M$-th link to the $m$-th link. Then, the total interference $I_{t}$ at Rx node $B$ is given as:

$$
I_{t}=I_{0}+\sum_{k=m}^{M} P_{C_{k}}\left|h_{B C_{k}}\right|^{2}
$$

where $I_{0}$ represents the total interference from the higherpriority links that are not currently under control, given as $I_{0}=\sum_{i=1}^{N} P_{A_{i}}\left|h_{A_{i} B}\right|^{2}$. Let $S I R_{A}^{\prime}$ denote the SIR adjusted by link $A-B$. Then, a corresponding channel capacity $R_{A}^{\prime}$ is given as:

$$
\begin{aligned}
R_{A}^{\prime} & =\log \left(1+S_{I R_{A}^{\prime}}\right) \\
& =\log \left(1+\frac{P_{A}\left|h_{A B}\right|^{2}}{I_{0}+\sum_{k=m}^{M} P_{C_{k}}\left|h_{B C_{k}}\right|^{2}}\right)
\end{aligned}
$$

Table 1 System model and simulation parameters

\begin{tabular}{cc}
\hline System parameters & Values \\
\hline System bandwidth $(W)$ & $5 \mathrm{MHz}$ \\
Slot duration $(T)$ & $2.08 \mathrm{~ms}$ \\
Maximum MS power $\left(P_{\max }\right)$ & $23 \mathrm{dBm}$ \\
Minimum MS power $\left(P_{\min }\right)$ & $3 \mathrm{dBm}$ \\
Power control step $(\Delta)$ & $1 \mathrm{~dB}$ \\
Buffer threshold $\left(\tau_{B}\right)$ & 1800 bytes \\
\hline Simulation parameters & Values \\
\hline Simulation area $(S)$ & $500 \times 500 \mathrm{~m}$ \\
Link distance $(d)$ & Uniform $[1,100] \mathrm{m}$ \\
Arrival rate $(\lambda)$ & 4 packets/slot duration \\
Packet length $(L)$ & 90 bytes \\
Path-loss exponent $(n)$ & 3.5 \\
\hline
\end{tabular}


Meanwhile, (4) must satisfy the bandwidth efficiency required for link $A-B, \mu_{A}$. If the maximum admissible interference is given as $I_{\max }$, the following constraint must be satisfied:

$$
\mu_{A} \leq R_{A}^{\prime}=\log \left(1+\frac{P_{A}\left|h_{A B}\right|^{2}}{I_{\max }}\right)
$$

From (5), therefore, the maximum admissible interference is computed as:

$$
I_{\max }=\frac{P_{A}\left|h_{A B}\right|^{2}}{2^{\mu_{A}}-1}
$$

In other words, the number of acceptable low-priority links is determined within the constraint of $I_{t} \leq I_{\max }$, from which a set of Tx nodes $\left\{C_{m *}, C_{m *+1}, \cdots, C_{M}\right\}$ can be accepted such that:

$$
m^{*}=\min \left\{\left.m\left|I_{\max } \geq I_{0}+\sum_{k=m}^{M} P_{C_{k}}\right| h_{B C_{k}}\right|^{2}\right\}
$$

The conservative yielding part is for the high-priority link to accept additional low-priority links while satisfying its own bandwidth efficiency requirement. In order to enable the conservative yielding part in practice, it must be ensured that the accepted low-priority links are not subject to Tx yielding. Recall that the Tx yielding part is governed by the inverse echo power of Tx node $B$, as illustrated in Figure 3. In other words, SIR measured at the Rx nodes that receive a CTS signal transmitted by Rx node $B$ with inverse echo power must be larger than the Rx yielding threshold. As the SIR measured at Rx node $B$ is given by the reciprocal of the inverse echo power $P_{E}$, the $\mathrm{Rx}$ node $B$ must adjust its CTS power so that the inverse echo power may be reduced. Such adjustment can be made by introducing a power control factor $\alpha$, which adjusts the inverse echo power as follows:

$$
P_{E}=\frac{\alpha}{P_{A}\left|h_{A B}\right|^{2}}
$$

where $0<\alpha<1$. As long as the low-priority nodes that receive the CTS signal with the inverse echo power in (8) satisfy the following condition:

$$
\frac{1}{\alpha} \cdot \frac{P_{A}\left|h_{A B}\right|^{2}}{P_{C_{m}}\left|h_{B C_{m}}\right|^{2}} \geq \eta_{T X}, m=M, M-1, \cdots, m^{*}
$$

they will be scheduled without $\mathrm{Rx}$ yielding. Since $\left|h_{B C_{1}}\right|^{2} \geq\left|h_{B C_{2}}\right|^{2} \geq \cdots \geq\left|h_{B C_{M}}\right|^{2}$, all inequalities in (9) can be satisfied with the power control factor $\alpha$ given as:

$$
\alpha^{*}=\frac{P_{A}\left|h_{A B}\right|^{2}}{P_{C}\left|h_{B C_{m *}}\right|^{2} \eta_{T X}}
$$

In other words, the Rx node of the high-priority link computes the inverse echo power by receiving an RTS signal over the tones in the Rx block, and then it transmits the CTS signal with the inverse echo power adjusted by the factor in (10). In order to determine the power adjustment factor in (10), a set of the low-priority links that can be accepted is determined by (7). A flowchart in Figure 6 summarizes the power control procedure for a CTS signal that implements the conservation yielding mechanism for the high-priority link.

\subsection{RTS power control for generous yielding}

The low-priority links that can be accepted by conservative yielding may still be subject to $\mathrm{Rx}$ yielding, which makes conservative yielding useless in practice. It is due to the situation that the received RTS signal of the low-priority link is weaker than the RTS interfer-

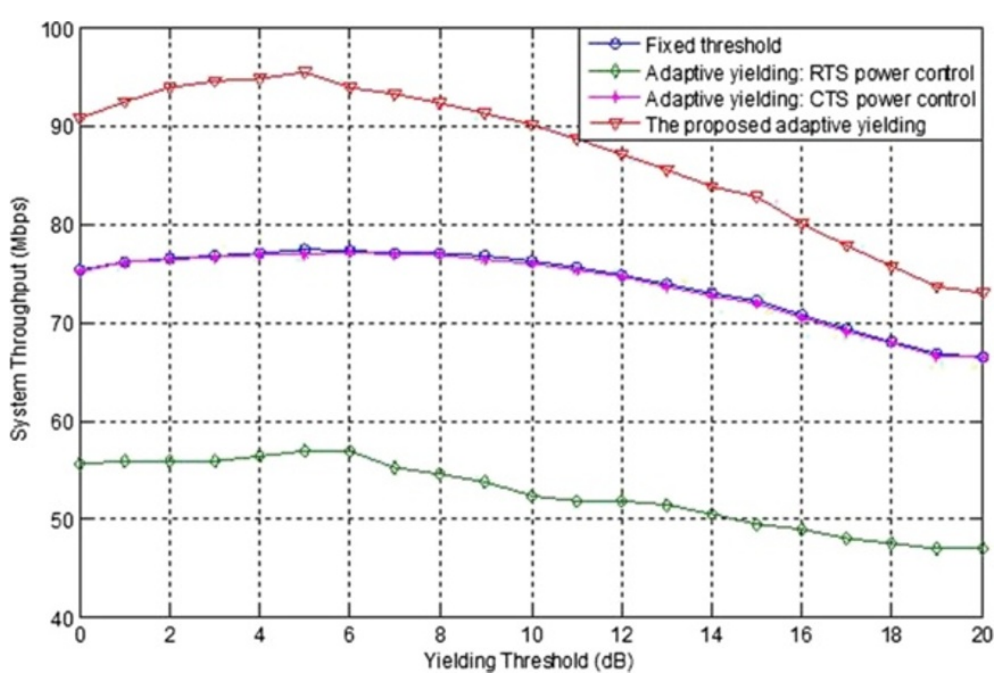

Figure 9 The average throughput performance as varying the yielding threshold $\left(\eta_{T X}=\eta_{R X}\right)$. 
ence signal of the high-priority link, which forces the low-priority link into Rx yielding even if it is exempted for Tx yielding by the conservative yielding mechanism in the high-priority links. In order to handle this situation, generous yielding by the high-priority links must be implemented.

The number of bits that are buffered in the transmitter of the high-priority link can be a basis to determine whether it yields or not by setting up a buffer threshold. In the case that the number of bits in the buffer is below the threshold, the transmit power can be reduced so that the low-priority links that are accepted by conservative yielding may not yield to the high-priority link. However, as it cannot be immediately known how generous it must be, we consider a step-by-step power control with a step size of $\Delta(\mathrm{dB})$. More specifically, its RTS transmit power is reduced by $\Delta$ for conservative yielding if the number of bits in the buffer exceeds the buffer threshold, or increased by $\Delta$ otherwise.

The node $A$ of the high-priority link in Figure 7 reduces its transmit power by a factor of $\beta$ for generous yielding, which ensures that the signal-to-interference-plusnoise ratio (SINR) in the receiver of the low-priority link exceeds the Rx yielding threshold as follows:

$$
\frac{P_{C_{m}}\left|h_{C_{m} D_{m}}\right|^{2}}{\beta \cdot P_{A}\left|h_{A D_{m}}\right|^{2}}>\eta_{R X}
$$

Of course, in the case that the number of bits exceeds the buffer threshold, the transmit node $A$ increases its power
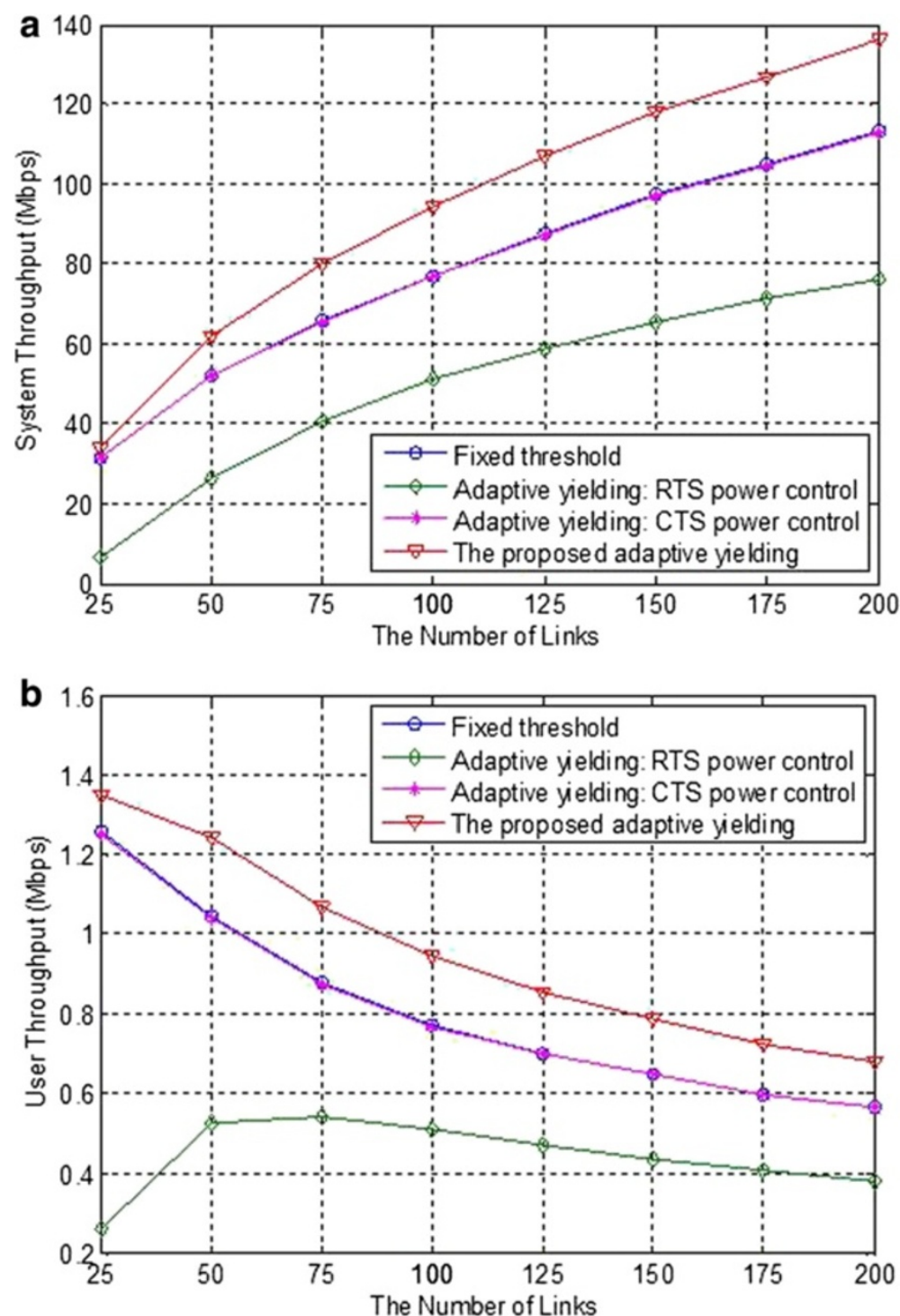

Figure 10 The average throughput performance: comparison. (a) Average system throughput. (b) Average per-user throughput. 
by a factor of $1 / \beta$ such that generous yielding is given up.

Figure 8 shows a flowchart of the RTS power control procedure for generous yielding. In this procedure, $P_{\max }$ and $P_{\min }$ represent the maximum and minimum transmit power, respectively, that can be set in the course of the power control process. Furthermore, $B$ and $\tau_{B}$ denote the number of bits waiting in the buffer and the buffer threshold, respectively. If $B>\tau_{B}$, the transmit power is increased by a step size of $\Delta(\mathrm{dB})$; otherwise, it is decreased by a step size of $\Delta(\mathrm{dB})$.

\section{Performance analysis}

In this section, we provide system-level simulation (SLS) results to analyze the performance gain of the proposed adaptive yielding scheme. We consider the FlashLinQ specification by Qualcomm Inc. as a baseline system model for our simulation, which is described in Section 2 [13]. We first describe a simulation scenario to evaluate the performance of the proposed adaptive yielding scheme and then present the simulation results to provide its performance gain over the conventional yielding scheme in FlashLinQ.

\subsection{Simulation scenario}

In this section, we provide system-level simulation (SLS) results to analyze the performance gain of the proposed adaptive yielding scheme. We consider the FlashLinQ specification by Qualcomm Inc. as a baseline system model for our simulation [13]. In order to focus on the performance of link-level scheduling only, we simply assume that synchronization, device discovery, and paging procedure for all links have been completed successfully. All yielding procedures with the pre-specified yielding thresholds are executed while randomly selecting the access priority for each link. The transmitters are uniformly located over a $500 \times 500\left(\mathrm{~m}^{2}\right)$ square area while locating the receiver in association with each transmitter at the distance uniformly selected from $[1,100](\mathrm{m})$. The packets arrive independently at each link by a Poisson distribution with an average arrival rate of $\lambda$ (packets/slot). For a channel model for each link, we consider the path loss only, which is represented as $P(d)$ for the distance $d$ between the transmitter and receiver. As the D2D communication link is shorter than a typical BSMS distance in the cellular system, it can be given in a path-loss model without a break point, i.e., $P(d)=d^{-n}$, where $n$ is a path-loss exponent. Table 1 summarizes the system model under consideration, including the simulation parameters. Unless stated otherwise, all the models and parameters in Table 1 are applied to the subsequent discussion.

\subsection{Simulation results}

In this subsection, we consider four different yielding schemes for performance analysis. The first scheme is the one with the fixed yielding thresholds, $\eta_{T X}$ and $\eta_{R X}$, which correspond to the existing scheme (denoted as 'fixed threshold'). Two other schemes are the adaptive ones with CTS power control for conservative yielding only (denoted as 'adaptive yielding: CTS power control') and RTS power control for generous yielding only (denoted as 'adaptive yielding: RTS power control'). The last scheme is an adaptive the proposed scheme that takes

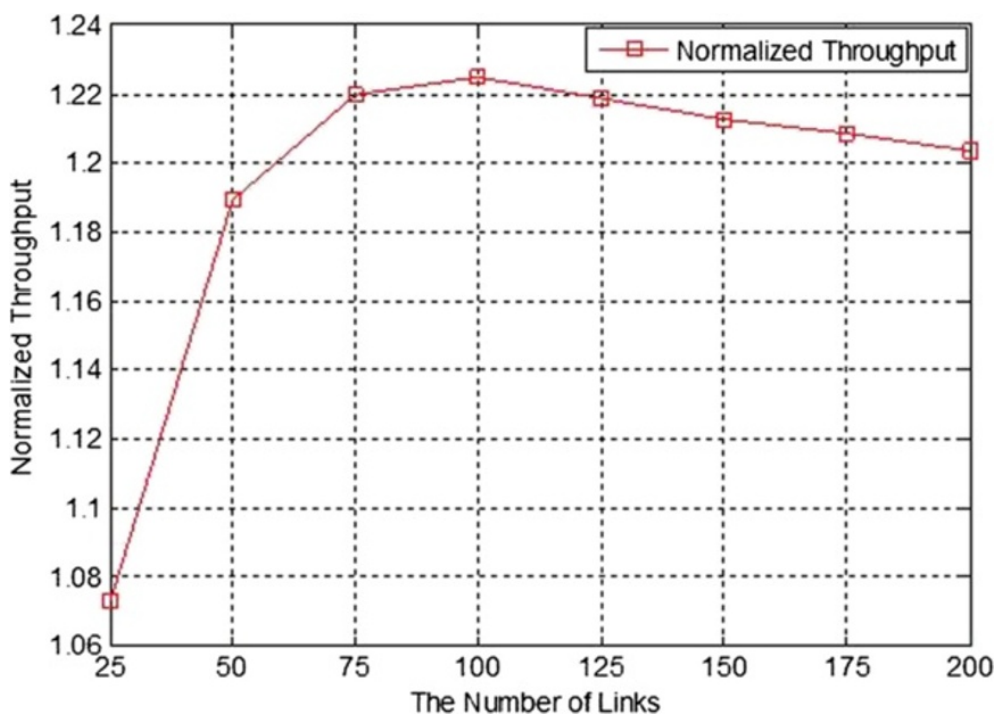

Figure 11 The normalized throughput gain of the adaptive yielding scheme over the conventional scheme. As varying the number of links. 
both conservative and generous yielding into account (denoted as 'the proposed adaptive yielding').

Figure 9 shows the average system throughput for the different schemes as varying the values of yielding threshold when the Tx and Rx yielding thresholds are set to be equal, i.e., $\eta_{T X}=\eta_{R X}$, and $100 \mathrm{D} 2 \mathrm{D}$ links are randomly distributed. We find that the performance is optimized roughly with $\eta_{T X}=\eta_{R X}=5 \mathrm{~dB}$ for all different schemes. Therefore, all simulation results throughout this paper are obtained by fixing the thresholds as $\eta_{T X}=\eta_{R X}=5 \mathrm{~dB}$.

Figure 10 shows the average system throughput and per-user throughput for the different schemes under consideration as the number of links is increased. In general, it is expected that the throughput performance increases with the link density, while its gain decreases gradually due to the co-channel interference among the links. The performance of the proposed adaptive yielding scheme always outperforms the yielding scheme with a fixed scheme, especially providing more gain with the higher link density.

Figure 11 shows the relative performance gain of the adaptive yielding scheme over that with the fixed threshold, providing a different gain as the link density varies. It shows that a maximum of $22.5 \%$ additional gain has been achieved by the proposed scheme in the current simulation scenario. Figure 10 and 11 show that a yielding scheme with only RTS or CTS power control does not provide any gain, which implies that the overall gain is mainly attributed to the integrated effect of both conservative and generous yielding in the proposed scheme.
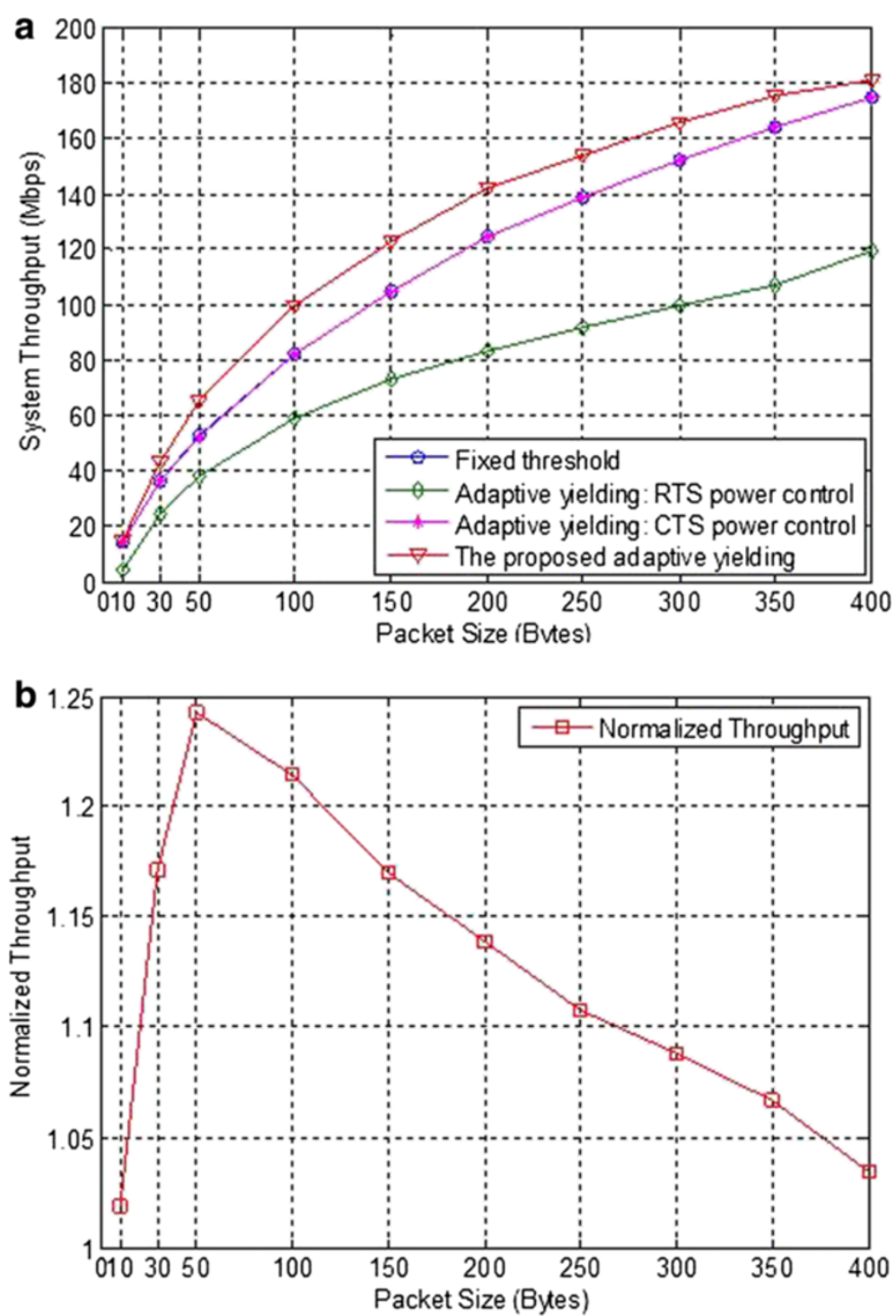

Figure 12 Average system throughput as varying the packet size. (a) Average system throughput. (b) Average per-user throughput. 

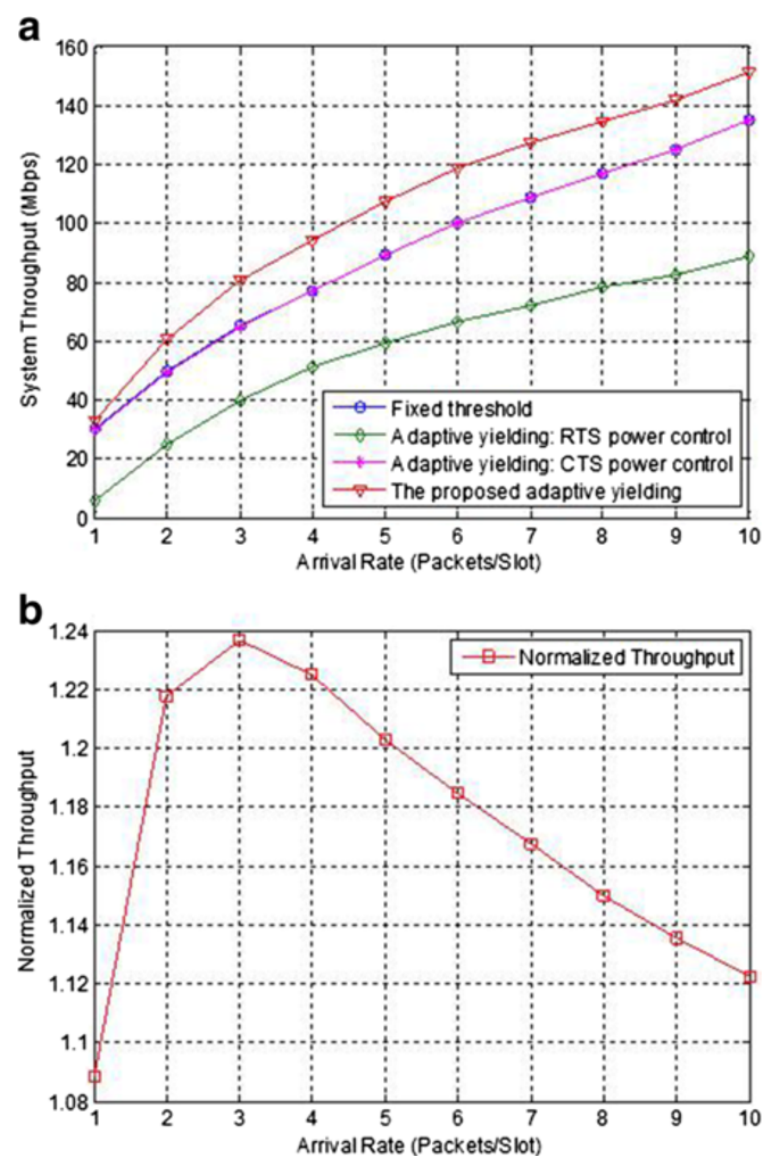

Figure 13 Average throughput performance as varying the packet arrival rate. (a) Average system throughput. (b) Average per-user throughput.

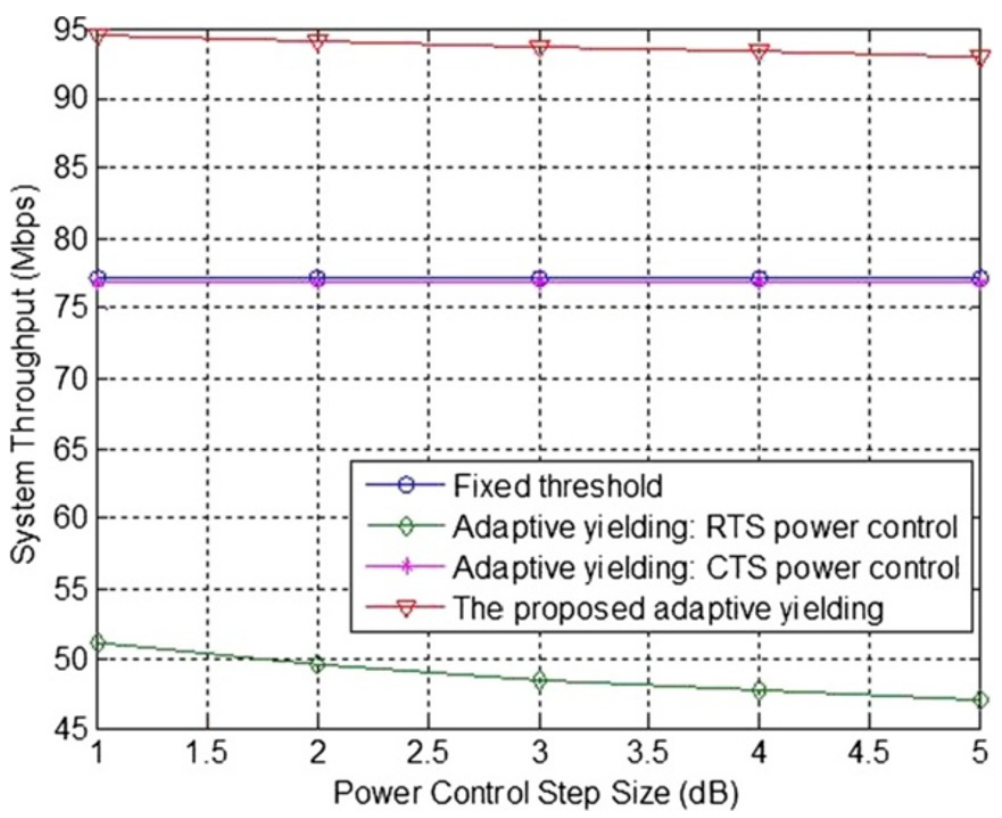

Figure 14 Average throughput performance as varying the power control step size $\Delta$. 
This can be explained by the observation that the yielding scheme with CTS power control only provides the same performance as one with the fixed threshold, implying that the additional low-priority link may not be scheduled by conservative yielding only. In other words, the proposed scheme would behave as designed only when both conservative and generous yielding are tightly integrated. Similarly, when only generous yielding is applied, the performance becomes worse than that of the original scheme (with the fixed threshold), as the RTS power is unnecessarily reduced.

Figure 12a shows the average system throughput for the different schemes as the packet length varies for the given number of links. As expected, the performance improves as the packet length increases, but it saturates when the effect of a spatial link reuse gain eventually disappears. It is clear that there is not much difference in the throughput performance between the yielding schemes with the fixed and adaptive thresholds when a packet size is too small. This is attributed to the fact that a short packet of the lowpriority link in the buffer will be immediately served even with a fixed threshold once it captures the channel by its random priority. Meanwhile, as the buffer size increases with the large packets, the opportunities of generous yielding becomes less frequent so as to make the throughput negligible, as observed in the normalized throughput gain in Figure 12b.

Figure 13a shows the average system throughput and relative gain for the different schemes with 100 links while varying the average packet arrival rate of $\lambda$ (packets/second). As expected, the system throughput increases with the average packet arrival rate, while it saturates eventually since traffic load is limited by the spatial reuse efficiency. Meanwhile, the opportunities of
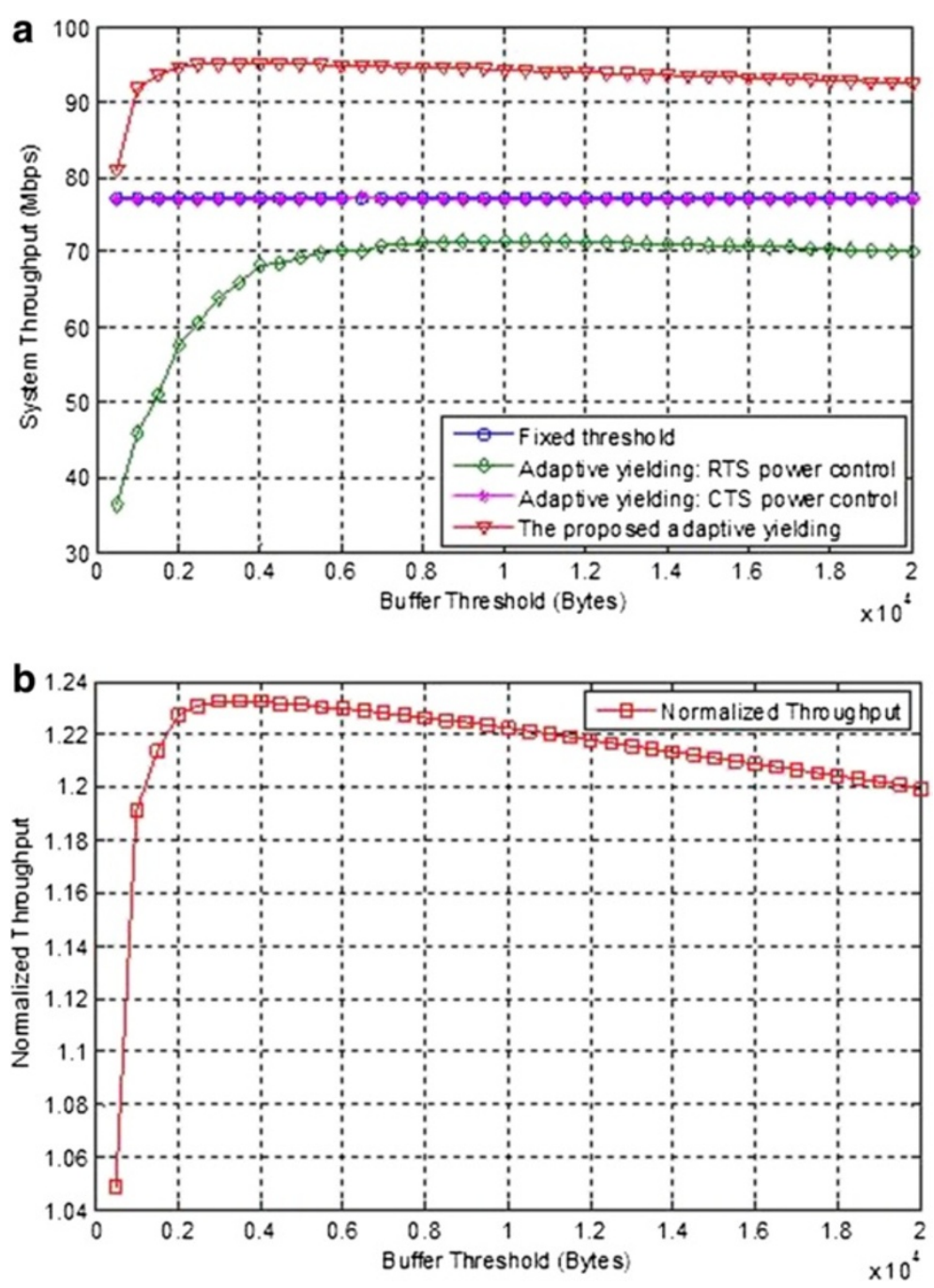

Figure 15 Average throughput performance as varying buffer threshold $\left(\boldsymbol{\tau}_{\boldsymbol{B}}\right)$. (a) Average system throughput. (b) Average per-user throughput. 
generous yielding disappear as the number of bits waiting in the buffer increases with the packet arrival rate (which is equivalent to the situation in which the packet size increases when the arrival rate is fixed), which ultimately reduces the throughput gain of the proposed scheme. When a packet arrival rate decreases, the gain is reduced as traffic load becomes smaller than the link capacity. As observed in the normalized throughput gain in Figure 13b, we find that there exists a traffic load that maximizes the performance of the proposed scheme, achieving the maximum gain of $24 \%$ over the existing scheme.

Figure 14 shows the average system throughput as the power control step size $\Delta$ is varied when considering 100 links with the packet length of $L=90$ bytes and $\lambda=4$ packets/slot. It is observed that the throughput performance slightly degrades as $\Delta$ increases. This implies additional performance degradation due to unnecessary yielding with an excessive step size, especially when it is now known how much generous yielding the low-priority link requires in practice. On the other hand, the proposed adaptive yielding scheme cannot work properly with insufficient step size, with which it may not execute generous yielding immediately.

Figure 15 shows the system throughput performance as the buffer threshold varies when there are 100 links with the packet length of $L=90$ bytes and arrival rate of $\lambda=4$ packets/slot. As shown in Figure 15b, there exists an optimal buffer threshold that maximizes the average throughput for the given traffic load. If the buffer threshold is too small, then adaptive yielding does not work since generous yielding is not applicable to most of links. Otherwise, the link capacity would be necessarily reduced as most links tend to reduce their transmit power. However, if the buffer threshold reaches a specific value, such as $\tau_{B}=1000$ bytes, the throughput performance dramatically increases and then remains almost constant even with a larger threshold. These observations are attributed to the fact that generous yielding is not incurred when the buffer threshold is too small, but it is incurred with stepby-step RTS power control when the buffer threshold is sufficiently large. Meanwhile, as the above performance may depend on the traffic load characteristics, such as the packet size and packet arrival rate, the buffer threshold must be set appropriately under the given traffic load.

\section{Conclusion}

In order to maximize the spatial resource reuse efficiency in the OFDM-based synchronous device-to-device (D2D) communication system, the number of devices that can maintain acceptable signal-to-interference ratio (SIR) in the receiver must be maximized. In this paper, we have introduced a notion of conservative yielding that allows for the low-priority link to be scheduled without overprovisioning the link quality of its proximate high-priority links with respect to the required bandwidth efficiency as a means of improving the throughput performance over the existing link scheduling scheme. We also have proposed an idea of generous yielding that allows for the low-priority link to be scheduled by falling back the data rate or delaying the transmission for a high-priority transmitter without too much data waiting in the buffer. We have tried to maximize the spatial reuse efficiency for the D2D link by integrating these concepts into the combined yielding scheme that adapts to the traffic load and link distribution. It has been demonstrated by system-level simulation that the proposed adaptive yielding scheme can improve the average throughput performance by more than $20 \%$ over the existing scheme with a fixed yielding threshold when the devices are uniformly distributed. As the actual performance gain mainly depends on the distribution of D2D links, its gain can be much higher under some other situations. In conclusion, the proposed link scheduling scheme can achieve more spatial reuse gain than the existing scheme, which has already packed the D2D links spatially as much as possible while providing fairness among the users by sharing the resources dynamically based on traffic demand. It is conjectured that the various types of yielding schemes considered in this paper can be applicable to different situations in which the bandwidth efficiency must be maximized by managing interference in actual traffic demand. For example, a similar concept can be applicable to operating wireless backhaul links in a wireless relay system in which the relay links must be reused while managing the inter-link interference in adaptation with the access traffic load in each relay.

\section{Competing interests}

The authors declared that they have no competing interests.

\section{Acknowledgements}

This work was supported in part by Communications Research Team, DMC R\&D Center, Samsung Electronics Co., Ltd. and also in part of the project titled Research on Fundamental Core Technology for Ubiquitous Shipping and Logistics funded by the Ministry of Oceans and Fisheries, Korea.

\section{Author details}

${ }^{1}$ Research Institute of Information and Communication Technology, Korea University, Seoul 136-701, South Korea. ${ }^{2}$ Department of Information and Communications Engineering, Dongguk University, Seoul 100-715, South Korea. ${ }^{3}$ School of Electrical Engineering, Korea University, Seoul 136-701, South Korea.

Received: 10 March 2014 Accepted: 9 September 2014 Published: 27 September 2014

\section{References}

1. Wi-Fi Alliance Specification: Wi-Fi Peer-to-Peer (P2P) Specification V1.1. (2011). Wi-Fi Alliance Specification

2. IEEE Std 802.15.1-2005: Wireless Medium Access Control (MAC) and Physical Layer (PHY) Specifications for Wireless Personal Area Networks (WPANs). (2005). IEEE Std 802.15.1-2005

3. IEEE Std 802.11-2007: Part 11: Wireless LAN Medium Access Control (MAC) and Physical Layer (PHY) Specifications. (2007). IEEE Std 802.11-2007 
4. K Koppler, M Rinne, C Wijting, C Ribeiro, K Hugl, Device-to-device communication as an underlay to LTE-advanced networks. IEEE Commun. Mag. 47(12), 42-49 (2009)

5. A Asadi, Q Wang, V Mancuso, A survey on device-to-device communication in cellular networks. (2013). arXiv preprint arXiv:1310.0720

6. B Wang, L Chen, X Chen, X Zhang, D Yang, Resource allocation optimization for device-to-device communication underlaying cellular networks, in Proceedings of IEEE Vehicular Technology Conference (VTC) (Budapest, 2011)

7. P Janis, V Kovunen, C Ribeiro, J Korhonen, K Doppler, K Hugl, Interference-aware resource allocation for device-to-device radio underlaying cellular networks, in Proceedings of IEEE Vehicular Technology Conference (VTC) (Barcelona, 2009)

8. S Hakola, T Chen, Device-to-device (D2D) communication in cellular network-performance analysis of optimum and practical communication mode selection, in Proceedings of IEEE Wireless Communication and Networking Conferenc (WCNC) (Australia, 2010)

9. P Janis, C Yu, K Doppler, C Ribeiro, C Wijting, K Hugl, O Tirkkonen, V Koivunen, Device-to-device communication underlaying cellular communications systems. Int'l. J. Commun. Netw. Syst. Sci. 2(3), 169-178 (2009). doi:10.4236/ijens.2009.23019

10. K Doppler, $\mathrm{CH} \mathrm{Yu,} \mathrm{Mode} \mathrm{selection} \mathrm{for} \mathrm{device-to-device} \mathrm{communication}$ underlaying an Ite-advanced network, in Proceedings of IEEE Wireless Communication and Networking Conferenc (WCNC) (Sydney, 2010)

11. TPeng, QX Lu, Interference avoidance mechanisms in the hybrid cellular and device-to-device systems, in Proceedings of IEEE International Symposium on Personal, Indoor and Mobile Radio Communications (PIMRC) (Tokyo, 2009)

12. T Chen, G Charbit, S Hakola, Time hopping for device-to-device communication in Ite cellular system, in Proceedings of IEEE Wireless Communication and Networking Conferenc (WCNC) (Sydney, 2010)

13. X Wu, S Tavildar, S Shakkottai, T Richardson, J Li, R Laroia, A Jovicic Flashlinq: A synchronous distributed scheduler for peer-to-peer ad hoc networks, in Proceedings of IEEE Allerton Conference on Communication, Control, and Computing (Allerton), (2010)

14. IEEE P802.16n/D1: Air Interface for Broadband Wireless Access Systems Draft Amendment: Higher Reliability Networks. (2012). IEEE P802.16n/D

15. IEEE P802.16.1a/D1: WirelessMAN-Advanced Air Interface for Broadband Wireless Access Systems Draft Amendment: Higher Reliability Networks. (2012). IEEE P802.16.1a/D1

16. N Naderializadeh, S Avestimehr, Itling: A new approach for spectrum sharing in device-to-device communication systems. (2014). arXiv preprint arXiv:1311.5527

17. IEEE Std. 802.11 b: Supplement to Part 11: Wireless LAN Medium Access Control (MAC) and Physical Layer (PHY) Specifications: Higher-speed Physical Layer Extension in the $2.4 \mathrm{GHz}$ Band. (1999). IEEE Std. 802.11b

18. F Ye, S Yi, B Sikdar, Improving spatial reuse of ieee 802.11 based ad hoc networks, in Proceedings of IEEE Global Communicatios Conference (GLOBECOM) (San Francisco, 2003)

19. S Sarkar, P Chaporkar, K Kar, Fairness and throughput guarantees with maximal scheduling in multi-hop wireless networks, in Proceedings of Modeling and Optimization in Mobile, Ad Hoc, and Wireless Network (WiOpt) (Boston, 2006)

20. J Monks, V Bharghavan, W Hwu, A power controlled multiple access protocol for wireless packet networks, in Proceedings of IEEE International Conference on Computer Communications (INFOCOM) (Anchorage, 2001)

21. VP Mhatre, K Papagiannaki, F Baccelli, Interference mitigation through power control in high density 802.11 wlans, in Proceedings of IEEE International Conference on Computer Communications (INFOCOM) (Anchorage, 2007)
22. CKToh, M Delwar, D Allen, Evaluating the communication performance of an ad hoc wireless network. IEEE Trans. Wireless Commun. 1(3) 402-414 (2002)

23. Y Xue, B Li, K Nahrstedt, Optimal resource allocation in wireless ad hoc networks: a price-based approach. IEEE Trans. Mobile Comput. 5(4), 347-364 (2006)

doi:10.1186/1687-1499-2014-157

Cite this article as: Kang et al:: Adaptive yielding scheme for link scheduling in OFDM-based synchronous device-to-device (D2D) communication system. EURASIP Journal on Wireless Communications and Networking 2014 2014:157.

\section{Submit your manuscript to a SpringerOpen journal and benefit from:}

- Convenient online submission

- Rigorous peer review

- Immediate publication on acceptance

- Open access: articles freely available online

- High visibility within the field

- Retaining the copyright to your article

Submit your next manuscript at $\boldsymbol{~ s p r i n g e r o p e n . c o m ~}$ 